\title{
New evidence on the origin of the microquasar GRO J1655-40
}

\author{
J. A. Combi ${ }^{1}$, L. Bronfman ${ }^{2}$, and I. F. Mirabel ${ }^{3,4}$ \\ 1 Departamento de Física (EPS), Universidad de Jaén, Campus Las Lagunillas s/n, 23071 Jaén, Spain \\ e-mail: jcombi@ujaen.es \\ 2 Departamento de Astronomía, Universidad de Chile, Casilla 36-D, Santiago, Chile \\ e-mail: leo@das.uchile.cl \\ 3 European Southern Observatory, Alonso de Cordova 3107, Santiago, Chile \\ ${ }^{4}$ On leave from CEA-Saclay, France \\ e-mail: fmirabel@eso.org
}

Received 24 January 2007 / Accepted 15 March 2007

ABSTRACT

\begin{abstract}
Aims. Motivated by the new determination of the distance to the microquasar GRO J1655-40 by Foellmi et al. (2006, A\&A, 457, 249), we conduct a detailed study of the distribution of the atomic (HI) gas, molecular (CO) gas, and dust around the open cluster NGC 6242, the possible birth place of the microquasar (Mirabel et al. 2002, A\&A, 395, 595). The proximity and relative height of the cluster on the galactic disk provides a unique opportunity to study SNR evolution and its possible physical link with microquasar formation.

Methods. We search in the interstellar atomic and molecular gas around NGC 6242 for traces that may have been left from a supernova explosion associated to the formation of the black hole in GRO J1655-40. Furthermore, the $60 / 100 \mu \mathrm{m}$ IR color is used as a tracer of shocked-heated dust.

Results. At the kinematical distance of the cluster the observations have revealed the existence of a HI hole of $1.5 \times 1.5$ in diameter and compressed $\mathrm{CO}$ material acumulated along the south-eastern internal border of the HI cavity. In this same area, we found extended infrared emission with characteristics of shocked-heated dust. Based on the morphology and physical characteristics of the HI, CO and FIR emissions, we suggest that the cavity in the ISM was produced by a supernova explosion occured within NGC 6242 . The lower limit to the kinematic energy transferred by the supernova shock to the surrounding interstellar medium is $\sim 10^{49}$ erg and the atomic and molecular mass displaced to form the cavity of $\sim 16.500 M_{\odot}$. The lower limit to the time elapsed since the SN explosion is $\sim 2.2 \times 10^{5} \mathrm{yr}$, which is consistent with the time required by GRO J1655-40 to move from the cluster up to its present position. The observations suggest that GRO J1655-40 could have been born inside NGC 6242, being one of the nearest microquasars known so far.
\end{abstract}

Key words. X-rays: individuals: GRO J1655-40 - ISM: bubbles - ISM: supernova remnants - radio lines: ISM - infrared: ISM

\section{Introduction}

Microquasars are X-ray binary systems exhibiting relativistic radio jets (Mirabel \& Rodríguez 1999). These systems contain compact objects like stellar black holes or neutron stars that accrete matter from a mass donor companion star. The formation of stellar black holes may result from direct collapse of massive star progenitors or from delayed collapse of proto-neutron stars that undergo energetic explosions (Mirabel 2004). In some cases, compact objects can suffer a strong natal kick imparted in the supernova event. Therefore, they can escape from the region of birth, where there could still exist signatures of their formation.

Until now, only 15 microquasars are known in the galaxy (Paredes 2005). Among them, GRO J1655-40 is one of the most studied ones, with observations available along the entire electromagnetic spectrum. The object is a binary system located at $(l, b)=\left(344^{\circ} .98,2^{\circ} .45\right)$, which contains a 5-7 $M_{\odot}$ black hole with a companion star of $2.3 M_{\odot}$ (Bailyn et al. 1995). Its origin has been discussed in the past in different frameworks. Israelian et al. (1999) detected large overabundances of $\mathrm{O}, \mathrm{Mg}$, $\mathrm{Si}$ and $\mathrm{S}$ in the atmosphere of the companion star, which were interpreted as evidence for supernova ejecta captured by the stellar atmosphere. The relative abundances of these elements suggest that the supernova progenitor could be a massive star with 25 to 40 solar masses.

Using radio continuum and $\mathrm{HI}$-line observations around the previously accepted distance of $3.2 \mathrm{kpc}$ for GRO J1655-40 (which corresponds to a velocity range from -42 to $-30 \mathrm{~km} \mathrm{~s}^{-1}$ ), Combi et al. (2001) found a cavity with "horse-shoe" morphology in the large-scale HI distribution. This kind of structure has been already observed towards early-type stars with very strong stellar winds (Benaglia \& Cappa 1999) and supernova remnants (Butt et al. 2001).

Other astrophysical connection between GRO J1655-40 and its environment has been suggested by Mirabel et al. (2002). These authors have used Hubble Space Telescope measurements to compute a runaway space velocity of $112 \pm 18 \mathrm{~km} \mathrm{~s}^{-1}$ for the object, which is moving in opposite direction of the open cluster NGC 6242, being the latter located at $(l, b)=\left(345^{\circ} .47,2.45\right)$. According to photometric and radial velocity measurements (Glushkova et al. 2006) this open cluster is located at a distance of $1.02 \pm 0.1 \mathrm{kpc}$ from the Sun. Therefore, such connection could not be confirmed since the old estimate of the distance for the microquasar had a value different from the one of the open cluster. Recently, the distance to GRO J1655-40 has been revisited by Foellmi et al. (2006). Using ESO archive and new VLT-UVES spectra they have determined a spectral type F6IV 
for the secondary star of the binary system. Thus, the distance to GRO J1655-40 would be smaller than $1.7 \mathrm{kpc}$. More recently, Foellmi (2006) showed that although this upper limit is rather firm, the lower limit is not well-constrained. Therefore, GRO J1655-40 could be one of the closest known black holes to the Sun.

In a recent paper on GRO J1655-40 by Sala et al. (2007) using XMM data the authors derive a link between the inner radius of the accretion disk and the distance. They argue that if the distance were smaller than $1.7 \mathrm{kpc}$ and the mass is $5 M_{\odot}$, then the inner accretion disk radius will be inside the gravitational radius of the black hole. However, the mass and spin of the black hole are uncertain. If the black hole were rapidly rotating as suggested by McClintock et al. (2006) and its mass less than $5 M_{\odot}$, the horizon of the Kerr black hole could be well inside the inner accretion radius. Furthermore, the authors admite that an estimate of the inner accretion radius using XMM data is model dependent.

Motivated by the new determination of the distance to the microquasar, we conduct here a detailed study of the distribution of the atomic (HI) and molecular (CO) gas, as well as of the dust in the vicinity of the open cluster NGC 6242. Our main goal is to detect the traces in the interstellar medium (ISM) around the cluster (i.e., a cavity), left by some explosive event. The proximity and relative height of the cluster on the galactic disk provides a unique opportunity to study SNR evolution and the possible physical link with microquasar formation. The paper is organized as follows: in Sect. 2 we describe our HI and CO observations. In Sect. 3 we present the main results and then we discuss the nature of the detected structures, and their possible physical connections with GRO J1655-40, in Sect. 4.

\section{2. $\mathrm{HI}$ and $\mathrm{CO}$ observations}

\subsection{HI observations}

We have performed HI observations towards GRO J1655-40 with a 30-m single dish telescope located at the Instituto Argentino de Radioastronomía (IAR), Villa Elisa, Argentina. The observations were carried out during six consecutive sessions on November 20-25, 2004. The radiotelescope has a helium-cooled HEMT amplifier with a 1008-channel autocorrelator, attaining a system temperature $T_{\text {sys }} \approx 35 \mathrm{~K}$. System parameters and additional details of the observational technique can be found in Combi et al. (1998). The HI line was observed in hybrid total power mode and the sky was sampled on a $0.35^{\circ}$ rectangular grid. Each grid position was observed during $60 \mathrm{~s}$ with a velocity resolution of $\sim 1 \mathrm{~km} \mathrm{~s}^{-1}$ and a coverage of $\pm 450 \mathrm{~km} \mathrm{~s}^{-1}$. A set of $\mathrm{HI}$ brightness temperature maps $\left(\Delta T_{\mathrm{rms}} \sim 0.2 \mathrm{~K}\right)$ were made for the velocity interval $\Delta v=\left(-80 \mathrm{~km} \mathrm{~s}^{-1},+20 \mathrm{~km} \mathrm{~s}^{-1}\right)$. The data were analyzed using AIPS routine programs.

\subsection{CO observations}

The ${ }^{12} \mathrm{CO}$ data were observed during 2002, October with the $4 \mathrm{~m}$ NANTEN telescope of Nogoya University, located then at Las Campanas (Carnegie Institution of Washington) in Chile. At the ${ }^{12} \mathrm{CO}(J=1-0)$ transition of $115.27 \mathrm{GHz}$, the angular resolution (HPBW) is 2 '.8. A field of $1.45 \times 1.45$ centered on $l=345^{\circ} .45$, $b=+2.5$ was observed in the ${ }^{12} \mathrm{CO}$ line with an integration time of $40 \mathrm{~s}$ for each position. The typical rms noise antenna temperature of the observations is in the range of $0.2-0.25 \mathrm{~K}$, and the velocity resolution is of $0.055 \mathrm{~km} \mathrm{~s}^{-1}$.

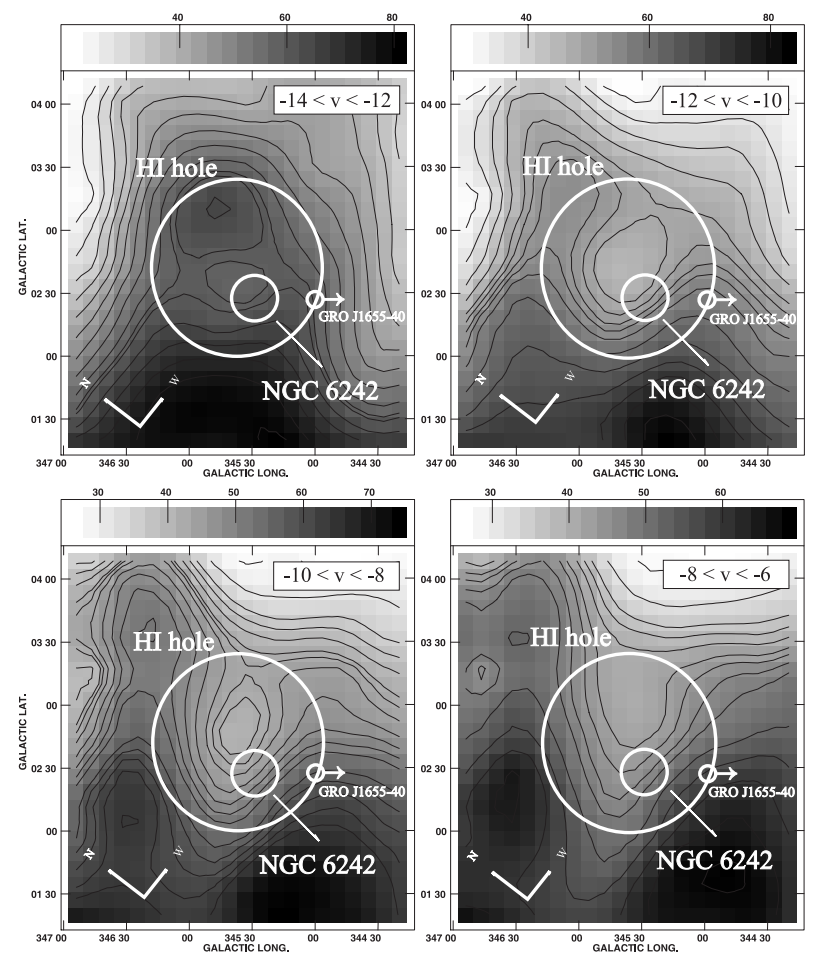

Fig. 1. HI brightness temperature channel maps obtained for the velocity range from -14 to $-6 \mathrm{~km} \mathrm{~s}^{-1}$ around the position of NGC 6242 (indicated with a white small circle). In all images the contours are labeled in steps of $4 \mathrm{~K}$, starting from $4 \mathrm{~K}$. A J2000 frame is shown for reference in the figure and all images. The location and motion direction of GRO J1655-40 are shown with a small circle and an arrow, respectively.

The data were reduced with the NANTEN data reduction software. Baselines were fitted to each individual spectrum. The resulting data cubes were transformed into FITS format, and further processing was done using the AIPS software.

\section{Main results}

If the physical link between GRO J1655-40 and NGC 6242 is real, the system should have been ejected from the cluster $\sim 3.4 \times 10^{5}$ years ago. After this time the radio emission of a SNR could be very faint or not detectable. In order to confirm this hypothesis, we search for signatures of some explosive event around NGC 6242 at radio frequencies using data from the 4.85-GHz (Condon et al. 1993) and 2.7-GHz surveys (Duncan et al. 1995), respectively. As expected, we found no traces of any SNR phenomena towards the region.

\subsection{Atomic component}

Standard galactic rotation models (Russeil 2003), corrected by excess associated with part of the Carina arm (Alvarez et al. 1990), indicate that for a kinematic distance in the range of 0.8 to $1.6 \mathrm{kpc}$ (the minimum distance to NGC 6242 and the maximun distance to GRO J1655-40), corresponds a velocity interval of -14 to $-6 \mathrm{~km} \mathrm{~s}^{-1}$. Figure 1, displays HI brightness temperature channel maps of the relevant interval. The development of a cavity centered at $(l, b)=\left(345.67^{\circ},+2.68^{\circ}\right)$ can be clearly seen in these maps. The angular size of this structure is $\sim 1.5 \times 1$. 5 . Thus, at an average distance of $1.2 \mathrm{kpc}$, the linear size of the HI structure would be $\sim 32 \mathrm{pc}$. The location of NGC 6242 and the HI cavitiy are indicated with white circles. 


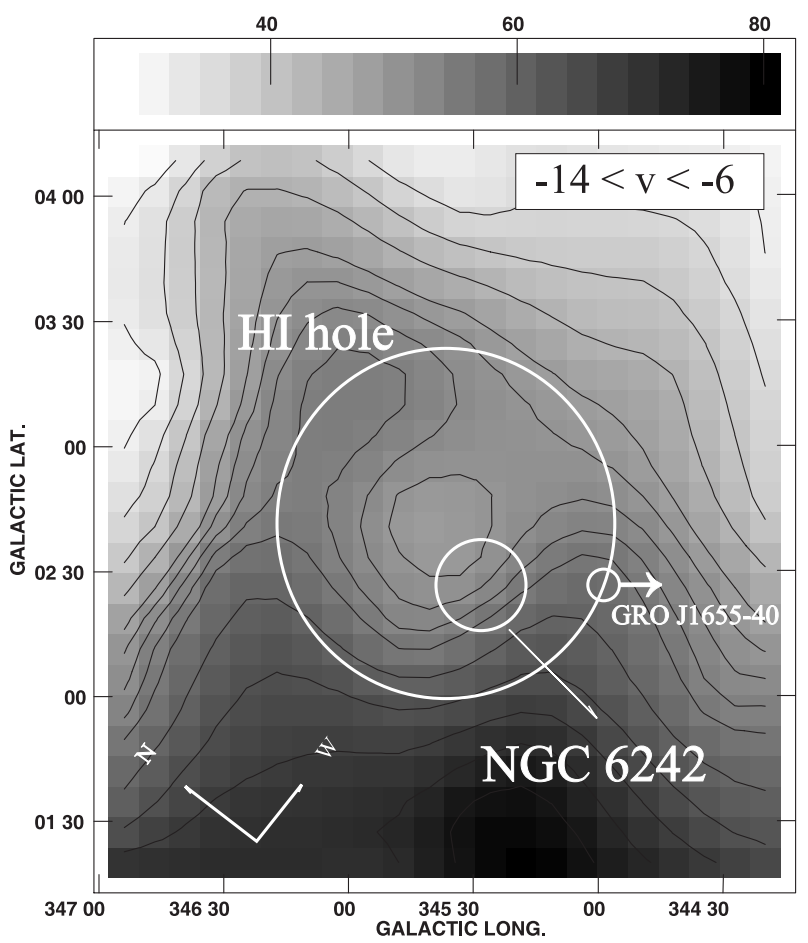

Fig. 2. Integrated column density map obtained for the velocity range from -14 to $-6 \mathrm{~km} \mathrm{~s}^{-1}$ around the position of NGC 6242 (indicated with a white circle). The contours are labeled in steps of $4 \mathrm{~K}$, starting from $4 \mathrm{~K}$. The location and motion direction of GRO J1655-40 are shown with a small circle and an arrow, respectively.

The angular extension of the cavity increases from -14 to $-10 \mathrm{~km} \mathrm{~s}^{-1}$. Between -12 to $-10 \mathrm{~km} \mathrm{~s}^{-1}$, the hole reaches its maximun size and forms a kind of "horse-shoe" structure at velocities larger than $-8 \mathrm{~km} \mathrm{~s}^{-1}$. The HI map integrated over the velocity interval from -14 to $-6 \mathrm{~km} \mathrm{~s}^{-1}$ is shown in Fig. 2.

Given that, no radio emission was detected towards the HI hole, we have computed the upper-limit for the radio surface brightness emission at $1 \mathrm{GHz}$. Flux densities at 2.7 and $4.8 \mathrm{GHz}$ of the region covered by the HI hole and an angular size for the cavity of $1.5^{\circ}$ were used. As may be expected, it turns out to be very low, with $\Sigma_{1 \mathrm{GHz}} \sim 4( \pm 2) \times 10^{-24} \mathrm{~W} \mathrm{~m}^{-2} \mathrm{~Hz}^{-1} \mathrm{sr}^{-1}$.

\subsection{Molecular component}

Figure 3 shows the distribution of the $\operatorname{CO}(J=1 \rightarrow 0)$ emission in a smaller area around NGC 6242 (indicated with a black circle). A large amount of compressed $\mathrm{CO}$ lies along the lower half of the image, in the region closer to the Galactic plane. This extended molecular feature coincides with the lower border of the HI cavity. It has a semi-circular morphology, typical of the interaction of a SNR shock with the steep density gradient towards the galactic plane. From -16 to $-6 \mathrm{~km} \mathrm{~s}^{-1}$ the angular extension of the cavity increases with the velocity. It is evident that in this velocity interval the molecular material was displaced to form part of the cavity seen in the HI distribution.

At the south region of the cluster the $\mathrm{CO}$ distribution exhibits a peculiar bifurcation. The morphology of the material could be the result from the eruption of a shock into a region of very low density. When the shock front hits a low density region it expands more rapidly into the interstellar medium producing a separation. The good agreement of the HI material, similar morphology and behavior of the molecular gas in this velocity range,
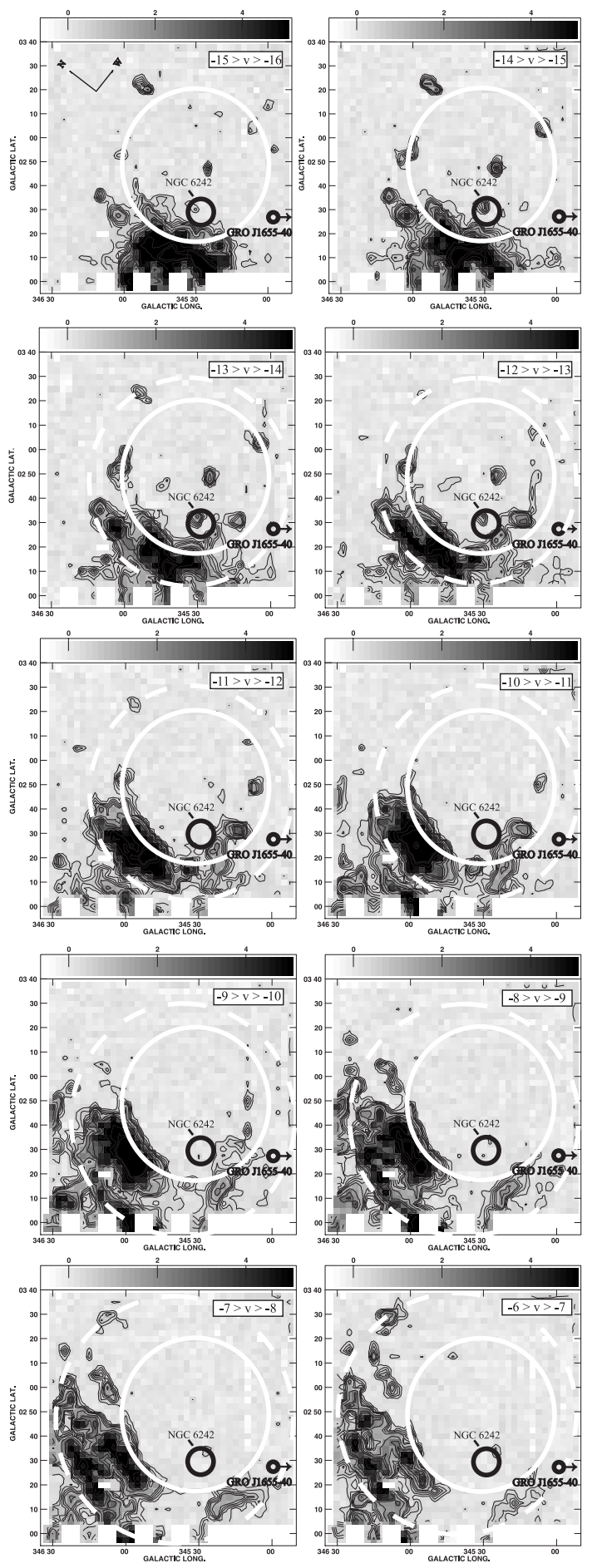

Fig. 3. The gray-scale channels map indicate the intensity of $\operatorname{CO}(J=$ $1 \rightarrow 0$ ) emission of the ambient molecular material, in the LSR velocity interval from -16 to $-6 \mathrm{~km} \mathrm{~s}^{-1}$ around the position of NGC 6242 (indicated with a black circle). White dashed circles show the expansion of the CO material along the velocity range. The location and motion direction of GRO J1655-40 are shown with a small circle and an arrow, respectively.

suggests that all these structures have a physical link and originate in the same explosive event.

A comparison of the $\mathrm{HI}$ distribution integrated in the velocity range from -14 to $-6 \mathrm{~km} \mathrm{~s}^{-1}$ with the ${ }^{12} \mathrm{CO}$ emission integrated in the same velocity range, is shown in Fig. 4. It is interesting to note that the molecular material is mainly displaced towards 


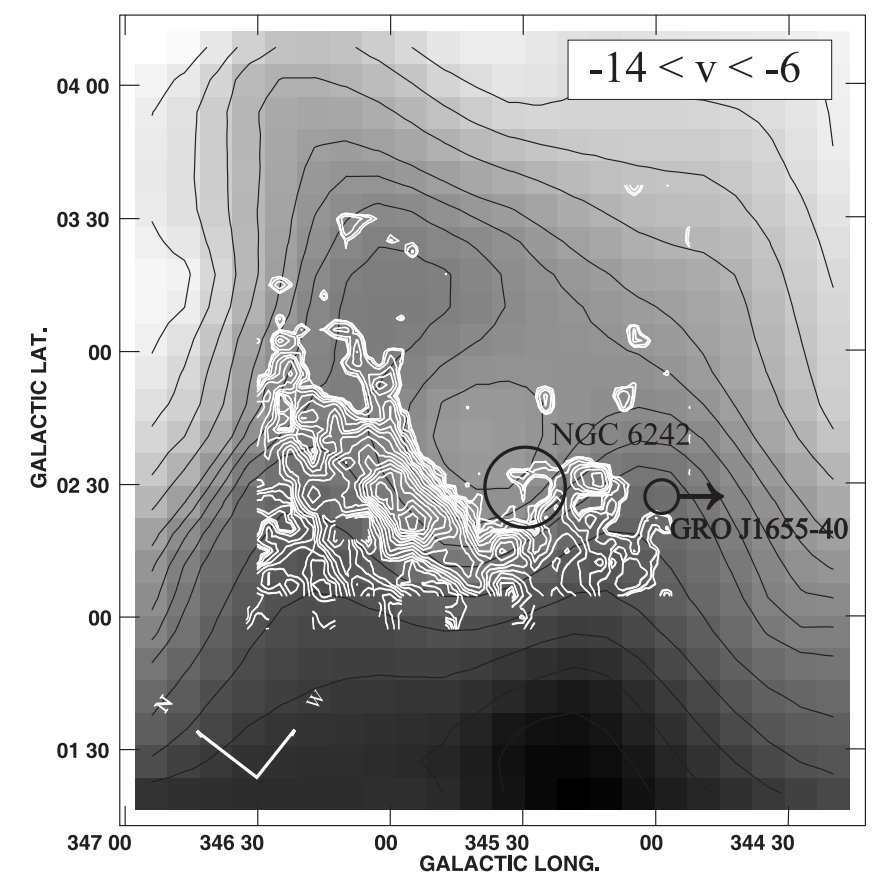

Fig. 4. Comparison of the HI distribution (in grey-scale) in the velocity range from -14 to $-6 \mathrm{~km} \mathrm{~s}^{-1}$ with the ${ }^{12} \mathrm{CO}$ emission integrated in the same velocity range. In the $\mathrm{HI}$ image the contour are labeled in steps of $4 \mathrm{~K}$, starting from $4 \mathrm{~K}$. The plotted ${ }^{12} \mathrm{CO}$ contours (white lines) are $2,2.5,3,3.5,6.5,9.5,16.5,25.5$ and $35.5 \mathrm{~K} \mathrm{~km} \mathrm{~s}^{-1}$. The location and motion direction of GRO J1655-40 are shown with a small circle and an arrow, respectively.

the lower region of the HI cavity. This is consequence of the fact that the mean thickness of the molecular disk is less than that of atomic gas (Bronfman et al. 1988). The circle indicate the position and aproximate extension of the open cluster NGC 6242.

\subsection{Infrared emission}

We have also searched for traces of a SNR shock using IRAS and $M S X$ observations. Figure 5 shows the MSX infrared emission at $8.28 \mu \mathrm{m}$ around NGC 6242 . The size of the image represents the HI hole shown in Fig. 2. In spite that is a very confuse region of the Galaxy, we found that several IR structures with extended morphology have good correlation with the molecular emission along the southern part of the image. At the central region of the HI hole we found extended IR emission with a semi-circular morphology (feature A). For all these structures the IR emission follows the spectral trend $F_{12} \leq F_{25} \leq F_{60} \leq F_{100}$ (where $F_{\lambda}$ is the flux density at wavelenght $\lambda$ ) characteristic of shockedheated dust (Junkes et al. 1992). At the southern part of the image, we can also see a large amount of IR emission, which could represent the shocked dust component of the associated CO material.

An increase in the $60 / 100 \mu \mathrm{m}$ IR color is a useful tracer of shocked-heated dust. The $60 / 100 \mu \mathrm{m}$ color-corrected ratio for the observed infrared emission of the whole region is shown in Fig. 6. It is interesting to note, that the infrared ratio level changes abruptly on the south border of the HI hole, which suggests the presence of the SNR shock. The small black circle represents the aproximate extension of NGC 6242. Enhanced IR emission on the central region and the southern part of the HI hole, is revealed by the ratio $60 / 100 \mu \mathrm{m}$. This values agree very

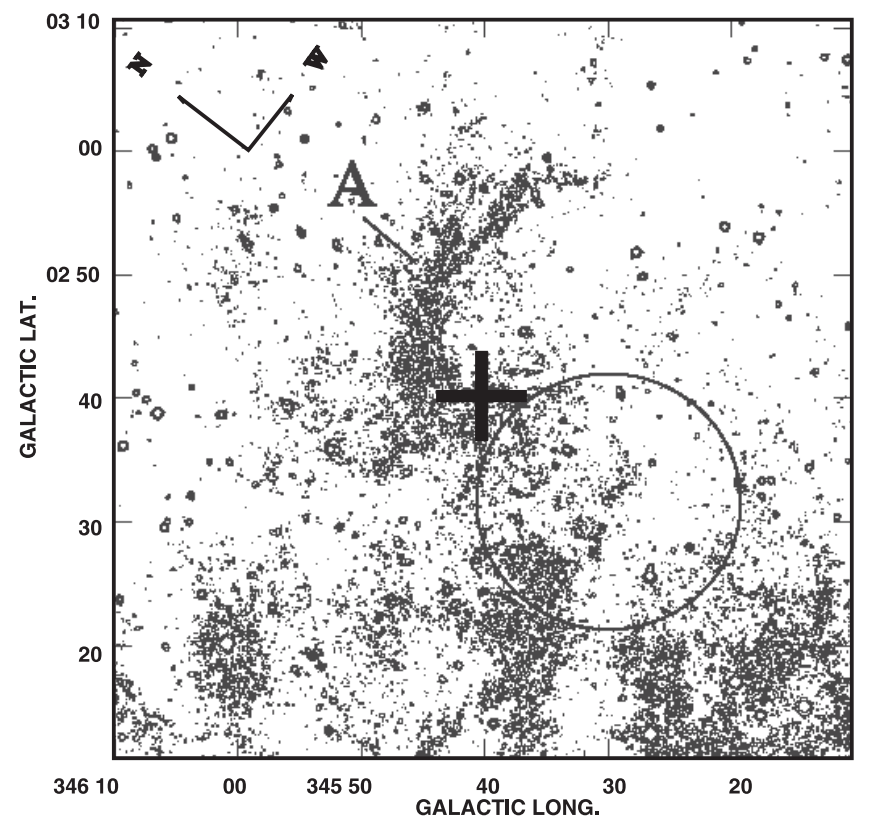

Fig. 5. Infrared emission from MSX at $8.28 \mu \mathrm{m}$. The position of the NGC 6242 is indicated with a black circle. The location of the center of the HI hole is indicate with a black cross. The size of the figure is approximately that of the $\mathrm{HI}$ cavity. The central semi-circular feature is labeled with letter A.

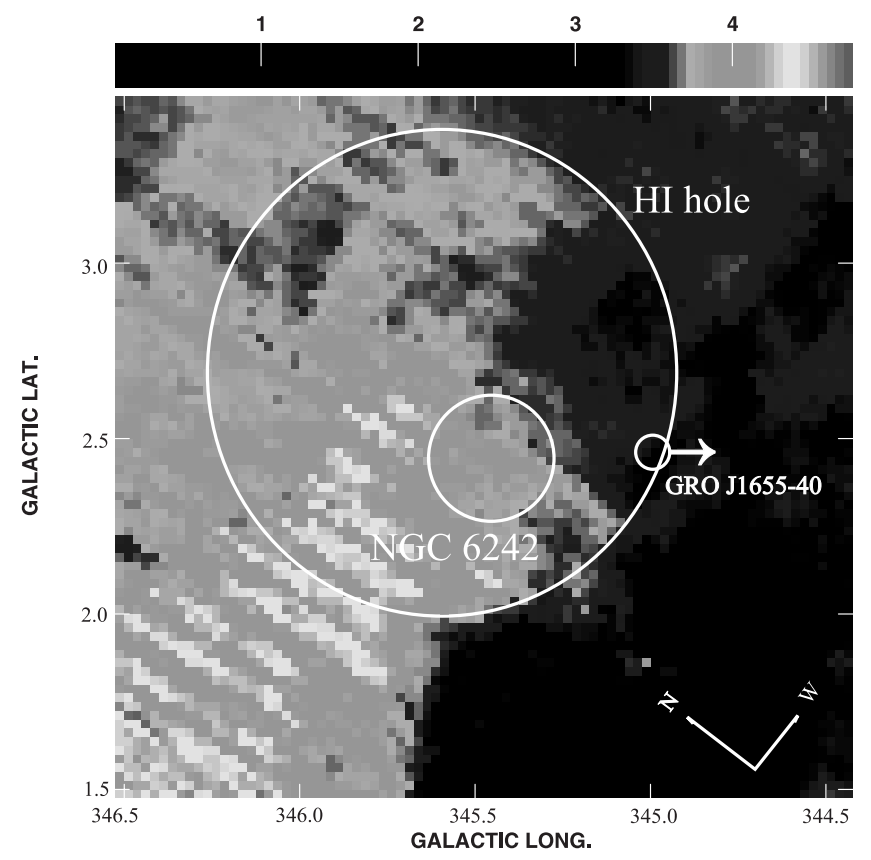

Fig. 6. Gray-scale distribution of the $60 / 100 \mu \mathrm{m}$ infrared ratio, which varies between 0.5 and 5 . Note as the infrared ratio level changes abruptly on the south border of the HI hole. The position of the HI hole and NGC 6242 are indicated with white circles. The location and motion direction of GRO J1655-40 are shown with a small circle and an arrow, respectively.

well with the color temperature derived for IRAS sources associated with shocked material in old SNRs (Huang et al. 1986).

\section{Discussion}

Atomic and molecular observations reveal that NGC 6242 is surrounded by shocked material with semi-circular structure, 
typical of a medium where a large amount of energy has been release. The existence of a local minimum in the HI distribution at the approximated position of the cluster is evident. It is confirmed by the $\mathrm{CO}$ distribution which is bending on the lower border of the HI cavity. Moreover, infrared emission found within and along the south-eastern border of the HI hole, has properties typical of dust heated by collisions with the postshock gas. All these facts suggest that the detected emission has an underlying physical connection originated in the same explosive phenomenon.

The energetic impact of a SN explosion that occurs out of the galactic disk can drive an asymmetric expanding bubble if the surrounded ambient gas is non-uniform. The shock-front could severely affect the dynamic structure of the surrounding gas, originating the observed semi-circular cavity, and heating the ambient dust. Assuming that the HI-CO cavity is the signature left by the action of a SNR on the surrounding ISM, we can use the N H I images and CO maps to estimate the mass sweptup by the front-shock. It allows to obtain the initial ambient gas density and roughly to estimate the time elapsed since the SN explosion.

Estimates of the physical parameters for the HI structure were derived by fitting a circle to the maximum of the envelope, which covers an area of $\Delta l \times \Delta b \approx 1.5 \times 1.5$. The integrated column densities shown in Fig. 2 were used to estimate the total mass displaced to form the cavity. If the background density is given by the density at the minimum, we obtain that at least $9.000 M_{\odot}$ have been removed from this region. The expansion velocity of the shell is defined approximately as $(\Delta v / 2)+2 \mathrm{~km} \mathrm{~s}^{-1}$, where $\Delta=v_{2}-v_{1}$ is the velocity interval through which the cavity is seen. Using an average expansion velocity of $\sim 12 \mathrm{~km} \mathrm{~s}^{-1}$, we obtain a lower limit to the original energy released in the explosion of $\sim 2 \times 10^{49} \mathrm{erg}$. Since the SNR can have lost a significant part of its energy via radiative cooling, the initial energy could have been $\sim 10^{51} \mathrm{erg}$. The mean density before the explosion (i.e. the initial density) can be obtained using the removed mass from the envelope's volume. Averaging the column densities within the cavity, we obtain that the density due to atomic material is $\sim 14 \mathrm{~cm}^{-3}$.

We further estimate the molecular component (H2) of the initial density, averaging 41 molecular clouds of the Carina arm taken from the study of Grabelsky et al. (1988). Taking into account the region of the lower half of the HI hole and including the correction of $\mathrm{He}$, we obtain that the density of molecular hydrogen would be $\sim 14 \mathrm{~cm}^{-3}$, that corresponds to $7500 M_{\odot}$ (which renders a total atomic and molecular displaced mass of $16.500 M_{\odot}$ ). Assuming that the whole cavity has been emptied of $\mathrm{HI}$ material and only the lower half of $\mathrm{CO}$, we estimate that an upper limit for the initial density, averaged over the whole cavity, is $N(\mathrm{H}) \sim 28 \mathrm{~cm}^{-3}$. With this density and a radius of $16 \mathrm{pc}$ for the cavity, we can estimate the lower limit for the SNR's age using the dynamical evolution equations for an expanding SNR, described by Koo \& Kang (2004). The non-detection of radio emission and size of the SNR's radius, suggest that it is well within the radiative phase. The transition to this phase in SNR evolution is often triggered when the blast wave interacts with dense atomic and molecular clouds of gas in the ISM, a fact supported by the large amount of shocked gas observed in the region.

According to Koo \& Kang (2004), the maximum age of the HI hole due to the SNR is defined as the time when the shock velocity drops to the ambient sound speed, so that the interior pressure is comparable to the ambient pressure and the shell breaks up. In this case, the lifetime of the SNR is described by:

$\tau=2.02 \times 10^{6}\left(\beta c_{\mathrm{s}, 6}\right)^{-1.43} n_{0}^{-0.367} E_{51}^{0.316} \mathrm{yr}-0.91 t_{\mathrm{sf}}$

where $c_{\mathrm{s}, 6}=c_{\mathrm{s}} /\left(10^{6} \mathrm{~cm} \mathrm{~s}^{-1}\right), c_{\mathrm{s}}$ is the sound speed, $\beta=2, E_{51}$ is the SN energy released to the ISM in units of $10^{51} \mathrm{erg}, n_{0}$ is the medium density, and $t_{\mathrm{sf}}$ is the shell formation time given by

$t_{\mathrm{sf}}=3.61 \times 10^{4} n_{0}^{-4 / 3} E_{51}^{3 / 14} \mathrm{yr}$.

Using a mean density of $n_{0} \sim 28 \mathrm{~cm}^{-3}$ and a SN energy of $\sim 10^{51} \mathrm{erg}$, we obtain $t_{\mathrm{sf}}=400 \mathrm{yr}$. For $c_{\mathrm{s}, 6}=1$, the lower limit to the lifetime of the SNR is $\tau=2.2 \times 10^{5} \mathrm{yr}$.

This value is on the order of the time elapsed by GRO J1655-40 in moving from NGC 6242 up to its present position, which is $\sim 3.4 \times 10^{5} \mathrm{yr}$.

\section{Summary}

We have investigated the surroundings of the open cluster NGC 6242 through HI, CO, and IR observations. Several facts corroborate the hypothesis of a $\mathrm{SN}$ event occurring within or very close to NGC 6242 .

- The HI brightness temperature channel maps for the distance range of $1.2 \pm 0.4 \mathrm{kpc}$ show the development of a cavity with an angular size of $\sim 1.5 \times 1.5$.

- The distribution of the $\mathrm{CO}(J=1-0)$ emission displays a large amount of compressed material with semi-circular morphology along the south and east borders of the HI cavity.

- Infrared observations reveal the presence of several IR structures with extended morphology and good correlation with atomic and molecular emissions. The 60/100 $\mu \mathrm{m}$ colorcorrected ratio shows also enhanced infrared emission towards the south-eastern part of the HI hole. These values agree very well with the color temperature derived for IRAS sources associated with shocked material in old SNRs.

- All the available information suggests that the detected emissions morphologies have a physical link, originated in a supernova explosion occurred within or very close to the cluster a $t \gtrsim 2.2 \times 10^{5}$ years. If the association between the open cluster and the SNR is confirmed then the distance of the microquasar would be better known.

Now, is the origin of GRO J1655-40 physically associated to the explosive event? We suggest that a possible scenario, which fits the data presented in this work, is that an old supernova explosion occurred at least $2.2 \times 10^{5}$ years ago inside NGC 6242. In this explosive event GRO J1655-40 could originate and consequently be ejected up to its present position in the sky. A similar case, not confirmed, could be that of the microquasar LS 5039 and the SNR G016.8-01.1 (Ribó et al. 2002).

Unveiling the origin of the microquasar will allow to constrain its distance. If the present evidence in this work is confirmed, GRO J1655-40 would be the first microquasar physically linked to a very dilute, but still detectable SNR.

High-resolution HI observations are required for a most detailed study of the cold gas and evidence of cloud compression originated by the shock front. Investigations of other molecular species, such as $\mathrm{OH}, \mathrm{HCO}+$ and $\mathrm{H} 2 \mathrm{CO}$, could help in search of shock signatures, particularly around the south-eastern region of the HI hole, where a large amount of gas and dust is detected.

Acknowledgements. We are very grateful to the referee for detailed suggestions which improved the paper significantly. We thank G. E. Romero and J. Martí for their critical reading of the manuscript. Remarks by T. Muñoz Darias are also 
acknowledge. J.A.C. is a researcher of the programme Ramón y Cajal funded jointly by the Spanish Ministerio de Educación y Ciencia (former Ministerio de Ciencia y Tecnología) and Universidad de Jaén. The authors also acknowledge support by DGI of the Spanish Ministerio de Educación y Ciencia under grants AYA2004-07171-C02-02 and AYA2004-07171-C02-01, FEDER funds and Plan Andaluz de Investigación of Junta de Andalucía as research group FQM322. This research made use of data products from the Midcourse Space Experiment. Processing of the data was funded by the Ballistic Missile Defense Organization with additional support from NASA Office of Space Science. This research has also made use of the NASA/IPAC Infrared Science Archive, which is operated by the Jet Propulsion Laboratory, California Institute of Technology, under contract with the National Aeronautics and Space Administration. L.B. acknowledges support from the Chilean Center for Astrophysics FONDAP 15010003.

\section{References}

Alvarez, H., May, J., \& Bronfman, L. 1990, ApJ, 348, 495

Bailyn, C. D., Orosz, J. A., McClintock, J. E., \& Remillard, R. A. 1995, Nature, 378,157

Benaglia, P., \& Cappa, C. E. 1999, A\&A, 346, 979

Bronfman, L., Cohen, R. S., Alvarez, H., et al. 1988, ApJ, 324, 248

Butt, Y. M., Torres, D. F., Combi, J. A., et al. 2001, ApJ, 562, L167
Combi, J. A., Romero, G. E., \& Arnal, E. M. 1998, A\&A, 333, 298 Combi, J. A., Romero, G. E., Benaglia, P., et al. 2001, A\&A, 370, L5 Condon, J. J., Griffith, M. R., \& Wright, A. E. 1993, AJ, 106, 1095 Duncan, A. R., Stewart, R. T., Haynes, R. F., et al. 1995, MNRAS, 277, 36 Foellmi, C., Depagne, E., Dall, T. H., et al. 2006, A\&A, 457, 249

Foellmi, C. 2006, Proc. of The Future of Photometric, Spectrophotometric and Polarimetric Standardization, ed. C. Sterken, ASP Conf. Ser.

Glushkova, E. V., Zabolotskikh, M. V., Rastorguev, A. S., et al. 1997, Astron. Lett., 23, 71

Grabelsky, D.A., Cohen, R.S., Bronfman, L., et al. 1987, ApJ, 315, 122 Grabelsky, D. A., Cohen, R. S., Bronfman, L., et al. 1988, ApJ, 331, 181 Junkes, N., Fuerst, E., \& Reich, W. 1992, A\&A, 261, 289

Huang, Y.-L., Dickman, R. L., \& Snell, R. L. 1986, ApJ, 302, L63 Israelian, G., Rebolo, R., Basri, G., et al. 1999, Nature, 401, 142 Koo, B.-C., \& Kang, J.-H. 2004, MNRAS, 349, 983

McClintock, J. E., Shafee, R., Narayan, R., et al. 2006, ApJ, 652, 518

Mirabel, I. F. 2004, Rev. Mex. Astron. Astrofis. Conf. Ser., 20, 14

Mirabel, I. F., \& Rodríguez, L. F. 1999, ARA\&A, 37, 409

Mirabel, I. F., Mignani, R., Rodrigues, I., et al. 2002, A\&A, 395, 595

Paredes, J. M. 2005, Chin. J. Astron. Astrophys., 5, 121

Ribó, M., Paredes, J. M., Romero, G. E., et al. 2002, A\&A, 384, 954

Russeil, D. 2003, A\&A, 397, 133

Sala, G., Greiner, J., Vink, J., et al. 2007, A\&A, 461, 1049 